\title{
Short hairpin RNA targeting AKT1 and PI3K/p85 suppresses the proliferation and self-renewal of lung cancer stem cells
}

\author{
AI-GUI JIANG ${ }^{1 *}$, HUI-YU LU ${ }^{1 *}$, DE-GENG ZHANG ${ }^{2}$, LI-XIN ZHANG ${ }^{3}$ and XIAO-YAN GAO ${ }^{1}$ \\ Departments of ${ }^{1}$ Respiratory Medicine and ${ }^{2}$ Oncology; ${ }^{3}$ Institute of Clinical Medicine, \\ Taizhou People's Hospital Affiliated to Medical College of Nantong University, Taizhou, Jiangsu 225300, P.R. China
}

Received May 16, 2014; Accepted February 9, 2015

DOI: $10.3892 / \mathrm{mmr} .2015 .3393$

\begin{abstract}
The aim of the present study was to investigate the effect of short hairpin (sh)RNA targeting AKT1 and phosphatidylinositol 3-kinase (PI3K)/p85 on the proliferation and self-renewal of lung cancer stem cells (LCSCs). The recombinant adenovirus expression vector, which contained shRNA targeting open reading frames of AKT1 and PI3K/p85, was transfected into LCSCs. It was found that AKT1 and PI3K/p85 expression was upregulated in LCSCs compared with that in the primary lung cancer cells. Recombinant adenovirus vector rAd5-siAKT1-siPI3K/p85 significantly downregulated AKT1 and $\mathrm{PI} 3 \mathrm{~K} / \mathrm{p} 85 \mathrm{mRNA}$ and protein expression in LCSCs. The downstream factors, proliferating cell nuclear antigen (PCNA) and cyclin D1 were also downregulated, while p53 was upregulated. Following silencing of AKT1 and PI3K/p85, cell proliferation, tumor sphere formation and tumor formation in NOD/SCID mice were also reduced. According to the present results, it was hypothesized that the PI3K/Akt signaling pathway is important in the self-renewal and proliferation of LCSCs, and that targeting the PI3K/Akt signaling pathway decreases the rate of tumor formation in vivo.
\end{abstract}

\section{Introduction}

Recent evidence has indicated that certain solid tumors, including brain gliomas $(1,2)$ and breast (3), prostate (4), colon (5) liver (6) and lung cancer (7) contain a small population of cancer stem cells (CSCs), which have a high capacity for self-renewal, multilineage differentiation, inducing malignancy, drug-resistance and radiotherapy resistance, as well as

Correspondence to: Dr Ai-Gui Jiang, Department of Respiratory Medicine, Taizhou People's Hospital Affiliated to Medical College of Nantong University, 210 Yingchun Road, Taizhou, Jiangsu 225300, P.R. China

E-mail: jiangaigui@126.com

*Contributed equally

Key words: lung cancer stem cells, lung cancer, AKT1, phosphatidylinositol 3-kinase/p85 recurrence and metastasis $(8,9)$. These cells are responsible for tumor maintenance and metastasis. It is postulated that therapies for cancer that specifically target stem cell signaling pathways utilized by CSCs may be beneficial $(10,11)$.

The phosphatidylinositol 3-kinase (PI3K)/Akt signaling pathway has been demonstrated to be involved in the regulation of cell proliferation and apoptosis, and is pivotal in the initiation and progression of malignancies, enhancing cell survival by stimulating cell proliferation and inhibiting apoptosis $(12,13)$. More recent studies have identified that the $\mathrm{PI} 3 \mathrm{~K} / \mathrm{Akt}$ signaling pathway is overactivated in several types of human cancer, including brain glioma (14), pancreatic cancer (15), lung cancer (16), and high expression of PI3K and p-Akt is often associated with a poor prognosis.

The overactivation of the PI3K/Akt signaling pathway has also been observed in several CSCs (17-19). However, there are no reports with regard to the correlation between the $\mathrm{PI} 3 \mathrm{~K} / \mathrm{Akt}$ signaling pathway, and proliferation and self-renewal of lung cancer stem cells (LCSCs) in the English-language literature. In the present study, a cell population with a CD133+ phenotype was isolated from the single cell suspension of lung adenocarcinoma tissue using a magnetic-activated cell sorting (MACS) technique, and enriched by serum free cultures. AKT1 and PI3K/p85 were suppressed by RNA interference. The expression of proliferating cell nuclear antigen (PCNA), cyclin D1 and p53 were also detected by western blot analysis. The effects of AKT1 and PI3K/p85 on the self-renewal and proliferation of LCSCs were investigated using an 3-(4,5-dimethylthiazol-2-yl)-2,5 -diphenyltetrazolium bromide (MTT) assay, sphere forming assay and xenograft formation assay. In addition, a cell cycle assay was also conducted using flow cytometry following AKT1 and PI3K/p85 silencing in LCSCs. The present study demonstrated that the PI3K/Akt signaling pathway is consistently overactivated in LCSCs. Additionally, it was revealed that the downregulation of AKT1 and $\mathrm{PI} 3 \mathrm{~K} / \mathrm{p} 85$ suppressed the self-renewal and proliferation of LCSCs and decreased the rate of tumor formation in vivo.

\section{Materials and methods}

LCSC isolation and cell culture. In our previous study (7), $\mathrm{CD}_{133}{ }^{+}$cells were successfully isolated from the single cell suspension of lung adenocarcinoma tissue using a MACS technique, and enriched by serum free culture. The sorting 
was verified with properties of LCSCs through experiments of self-renewal, multipotential differentiation capacity, drug resistance and tumorigenic capacity in vivo. LCSCs were harvested and cultured in serum-free Dulbecco's modified Eagle's medium (DMEM)-F12 (Beijing Beiruo Biothechnology Co., Ltd., Beijing, China) containing $50 \mu \mathrm{g} / \mathrm{ml}$ insulin (Sigma-Aldrich, St. Louis, MO, USA), $100 \mu \mathrm{g} / \mathrm{ml}$ apo-transferrin (SigmaAldrich), $10 \mu \mathrm{g} / \mathrm{ml}$ putrescine (Sigma-Aldrich), $0.03 \mathrm{mM}$ sodium selenite (Sigma-Aldrich), $2 \mu \mathrm{M}$ progesterone (Pure Chemistry Scientific, Inc., Sugarland, TX, USA), $0.6 \%$ glucose (LGM Pharma, Nashville, TN, USA), 5 mM HEPES (Nanjing Search Biotech Co., Ltd., Nanjing, China), $0.1 \%$ sodium bicarbonate, $0.4 \%$ bovine serum albumin (BSA; Wuhan Boster Biological Engineering Co., Ltd., Wuhan, China), glutamine (Ameresco, Inc., Solon, OH, USA) and 1\% penicillin and $1 \%$ streptomycin (Beijing Beiruo Biothechnology Co., Ltd.), as well as $20 \mathrm{ng} / \mathrm{ml}$ epidermal growth factor (EGF; PeproTech, Rocky Hill, NJ, USA) and $10 \mathrm{ng} / \mathrm{ml}$ basic fibroblast growth factor (bFGF; PeproTech) at $37^{\circ} \mathrm{C}$ and $5 \% \mathrm{CO}_{2}$.

Generation of rAd5-small interfering (si)AKT1-siPI3K/p 85 RNAi lentiviruses (Wuhan Boster Biological Engineering Co., Ltd.) After testing knockdown efficiencies of several shRNA constructs, the following shRNA oligonucleotides (rAd5-siAKT1-siPI3K-shRNA) were utilized: 5'-GGAGATCATGCAGCATCGC-3' [to target the 19 bp interference sequence of AKT1 gene (intervention sites: 1540-1558)] and 5'-GAAAGGAGGAAATAACAAA-3' [to target the 19 bp interference sequence of PI3K/p85 gene (intervention sites: 371-389]. A non-specific shRNA (rAd5-siCtrl-shRNA) was also synthesized as a control, and each shRNA was cloned into a pGCL-GFP plasmid containing the U6 promoter and green fluorescent protein (GFP). The plasmids pDONR221 and $\mathrm{pAD} / \mathrm{CMV} / \mathrm{V} 5-\mathrm{DEST}$ were also included to provide the necessary packaging elements for lentivirus production. For viral transduction, shRNA lentiviral vectors at a multiplicity of infection (MOI) of 25 were added to dispersed LCSCs rapidly following plating. GFP fluorescence was measured $72 \mathrm{~h}$ post-transduction.

Reverse transcription-quantitative polymerase chain reaction $(R T-q P C R)$. The primer sequences used included: Forward: 5'-GGCCCAGATGATCACCATCAC-3' and reverse: 5'-CTATCGTCCAGCGCAGTCCA-3' for AKT1; forward: 5'-AGCATTGGGACCTCACATTACACA-3' and reverse: 5'-ACTGGAAACACAGTCCATGCACATA-3' for PI3K/p85; and forward: 5'-CCTGGCACCCAGCACAAT-3' and reverse: 5'-GCCGATCCACACGGAGTACT-3' for $\beta$-actin. The total RNA was isolated using TRIzol reagent (Invitrogen Life Technologies) according to the manufacturer's instructions. cDNA was synthesized using a reverse transcription kit (Toyobo Co., Ltd., Osaka, Japan), according to the manufacturer's instructions. cDNA $(2.5 \mu 1)$ was subjected to qPCR using SYBR-Green as a fluorescent reporter and 2.5X Real Master mix (Toyobo, Osaka, Japan). Specific gene primers (Wuhan Boster Biological Engineering Co., Ltd.) for AKT1, $\mathrm{PI} 3 \mathrm{~K} / \mathrm{p} 85$ and $\beta$-actin, were amplified in separate reaction tubes. Threshold cycle numbers of triplicate reactions were determined using ABI-7500 software (v2.0; Invitrogen Life Technologies, Carlsbad, CA, USA) and averaged. Relative fold changes were calculated using the $2^{-\Delta \Delta C t}$ method and standard curves were produced.

Western blot analysis. Cell extracts from the control group (untreated cells), Ad5-Control-shRNA group (cells infected with Ad5-Control-shRNA) and Ad5-siAKT1-siPI3K-shRNA group (cells infected with Ad5-siAKT1-siPI3K-shRNA), as well as primary lung cancer cells were collected, and protein samples $(50 \mu \mathrm{g})$ were then electrophoresed on $12 \%$ SDS-polyacrylamide gels and transferred onto polyvinylidene fluoride (PVDF) membranes (Wuhan Boster Biological Engineering Co., Ltd.). Membranes were blocked in 5\% non-fat dry milk in Tris-buffered saline (TBS) for $1 \mathrm{~h}$ at room temperature and incubated with anti-AKT1 (sc-514032), anti-PI3K/p85 (sc-131324), anti-PCNA (sc-71858), anti-cyclin D1 (sc-70899) and anti-P53 (sc-377567) antibodies (diluted 1:1,000, Santa Cruz Biotechnology Inc., Santa Cruz, CA, USA) overnight at $4^{\circ} \mathrm{C}$. Membranes were incubated with $50 \mu \mathrm{lgG} /$ horseradish peroxidase secondary antibody (diluted 1:2,000, Wuhan Boster Biological Engineering Co., Ltd. Wuhan, China) for $2 \mathrm{~h}$ at room temperature after three washes with TBS-Tween-20 (TBST). Each membrane was also incubated with an anti- $\beta$-actin antibody (Santa Cruz Biotechnology Inc.) as a loading control. Membranes were washed three times with TBST and bound antibodies were detected using enhanced chemiluminescence (Beyotime, Jiangsu, China). Protein levels were quantitated by densitometry using Quantity One 4.62 software (Bio-Rad Laboratories, Munich, Germany).

Sphere-forming assay. Cell extracts from the control group, Ad5-Control-shRNA group and Ad5-siAKT1-siPI3K-shRNA group were dissociated and cultured in 96-well plates in serum-free DMEM-F12 medium containing $50 \mu \mathrm{g} / \mathrm{ml}$ insulin, $100 \mu \mathrm{g} / \mathrm{ml}$ apo-transferrin, $10 \mu \mathrm{g} / \mathrm{ml}$ putrescine, $0.03 \mathrm{mM}$ sodium selenite, $2 \mu \mathrm{M}$ progesterone, $0.6 \%$ glucose, $5 \mathrm{mM}$ HEPES, $0.1 \%$ sodium bicarbonate, $0.4 \%$ BSA, glutamine and $1 \%$ penicillin and $1 \%$ streptomycin, as well as $20 \mathrm{ng} / \mathrm{ml} \mathrm{EGF}$ and $10 \mathrm{ng} / \mathrm{ml} \mathrm{bFGF}$. Wells containing more than one cell or no cells were marked and dismissed from the statistical data. The cells were cultured under conditions of $5 \% \mathrm{CO}_{2}$ at $37^{\circ} \mathrm{C}$ for 10 days. The medium was replaced or supplemented with fresh growth factors twice a week. Wells that contained spheres were counted using inverted phase contrast microscopy (DMILPH1; Leica, Mannheim, Germany) and the percentage of cells with sphere-forming capacity was calculated.

Proliferation assay. The proliferation of the cells was detected using an MTT assay on days 1, 3, 5 and 7. Three groups were plated onto 96 -well plates $(2,000$ cells in $0.2 \mathrm{ml}$ cell culture medium/well). The cells were then incubated with $20 \mu 1 \mathrm{MTT}$ ( $5 \mathrm{mg} / \mathrm{ml}$; Sigma-Aldrich, St. Louis, MO, USA) for $4 \mathrm{~h}$ prior to collection. The culture medium was finally removed, and $150 \mu \mathrm{l}$ dimethylsulfoxide was added to the well. After shaking thoroughly for $10 \mathrm{~min}$, the plates were read for absorbance in an enzyme immunoassay at $490 \mathrm{~nm}$ using an Automatic enzyme-linked immunity analyzer (Diasorin S.p.A, Italy). Six wells were analyzed for each group.

Cell cycle phase distribution. A total of $1 \times 10^{6}$ cells from the control group, Ad5-Control-shRNA group and 

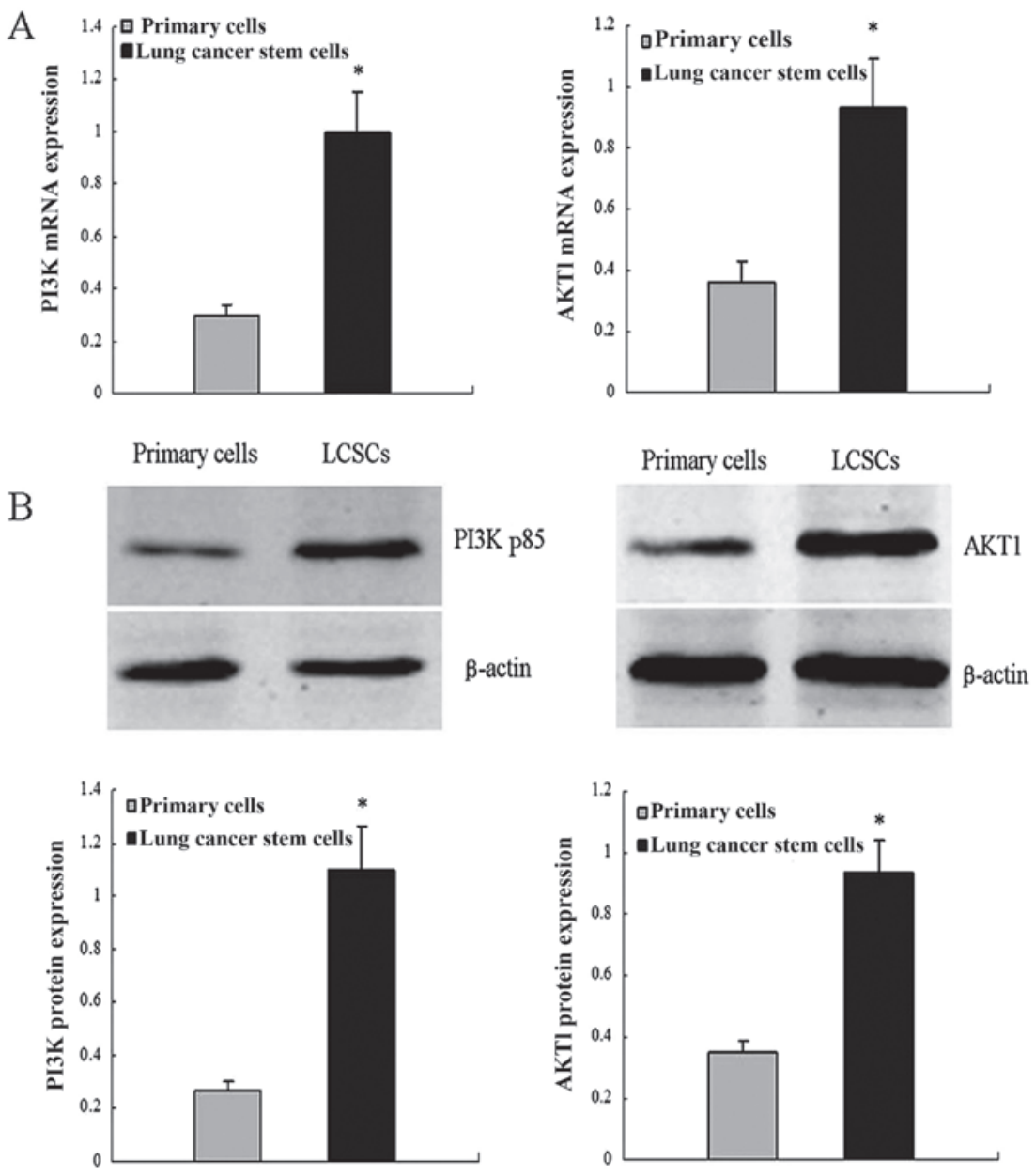

Figure 1. mRNA and protein expression of PI3K and AKT1. (A) Reverse transcription-quantitative polymerase chain reaction assays revealed that AKT1, $\mathrm{PI} 3 \mathrm{~K} / \mathrm{p} 85$ mRNA abundance was higher in LCSCs compared with primary lung cancer cells. ("P $<0.01, \mathrm{n}=3$ ). (B) Similar results from western blot assays using the same cell types confirmed this finding. ("P<0.01, $\mathrm{n}=3$ ). PI3K, phosphatidylinositol 3-kinase; LCSCs, lung cancer stem cells.

Ad5-siAKT1-siPI3K-shRNA group were centrifuged at $375 \mathrm{x}$ g for $5 \mathrm{~min}$, resuspended in $0.2 \mathrm{ml}$ phosphate-buffered saline and then fixed in $1 \mathrm{ml}$ of $70 \%$ ethanol at $4^{\circ} \mathrm{C}$ for $16 \mathrm{~h}$. Subsequent to washing with PBS, the cells were incubated with $300 \mu \mathrm{l}$ Green-DNA Dye (Nanjing Search Biotech Co., Ltd., Nanjing, China) at room temperature for $30 \mathrm{~min}$. Cell cycle status was assessed by flow cytometry (Beckman Coulter, Brea, CA, USA). The relative proportions of cells in the $G_{0} / G_{1}$, $\mathrm{S}$ and $\mathrm{G}_{2} / \mathrm{M}$ phases were analyzed, and the percentages of cells in each phase were calculated.

TumorigenicityinNOD/SCIDmice.Cellsfrom the controlgroup, Ad5-Control-shRNA group and Ad5-siAKT1-siPI3K-shRNA group were diluted in growth factor-containing medium alone prior subcutaneous injection. Serial dilutions of cells $\left(10^{2}\right.$, $10^{3}, 10^{4}$ and $10^{5}$ cells) were injected subcutaneously into the abdominal wall of 4-week-old NOD/SCID mice (5 mice/group; Beijing Vitalriver Experimental Animal Technical Co., Ltd, Beijing, China). Tumor size was measured using calipers and tumor volume was calculated using the equation $V=\pi / 6$ (length $\mathrm{x}$ width $\mathrm{x}$ height). The mice were sacrificed 8 weeks following the subcutaneous injection. The present animal study was approved by the Ethics Committee of Taizhou People's Hospital (Jiangsu, China) and was performed according to the Declaration of Helsinki.
Statistical analysis. Statistical analysis was performed using SPSS 13.0 software (SPSS, Inc., Chicago, IL, USA). All experiments were performed at least three times and representative results are presented as the mean \pm standard deviation. Statistical analysis was performed by one-way analysis of variance and comparisons among groups were achieved using independent sample t-tests. $\mathrm{P}<0.05$ was considered to indicate a statistically significant difference.

\section{Results}

AKT1 and PI3K/p85 expression in primary lung cancer cells and LCSCs. RT-qPCR assays were performed to detect AKT1 and PI3K/p85 mRNA levels. In these assays, AKT1 and PI3K/p85 mRNA abundance was higher in LCSCs compared with the primary lung cancer cells. Similar results from western blot assays using the same cell types confirmed the results. These results indicated that AKT1 and PI3K/p85 expression is upregulated in LCSCs (Fig. 1).

Efficiency of adenovirus transfection. For viral transduction, shRNA lentiviral vectors at an MOI of 25 were added to dispersed LCSCs shortly following plating. After three days, the GFP expression of the LCSCs confirmed that the lentiviral infection was achieved, compared with the corresponding white 

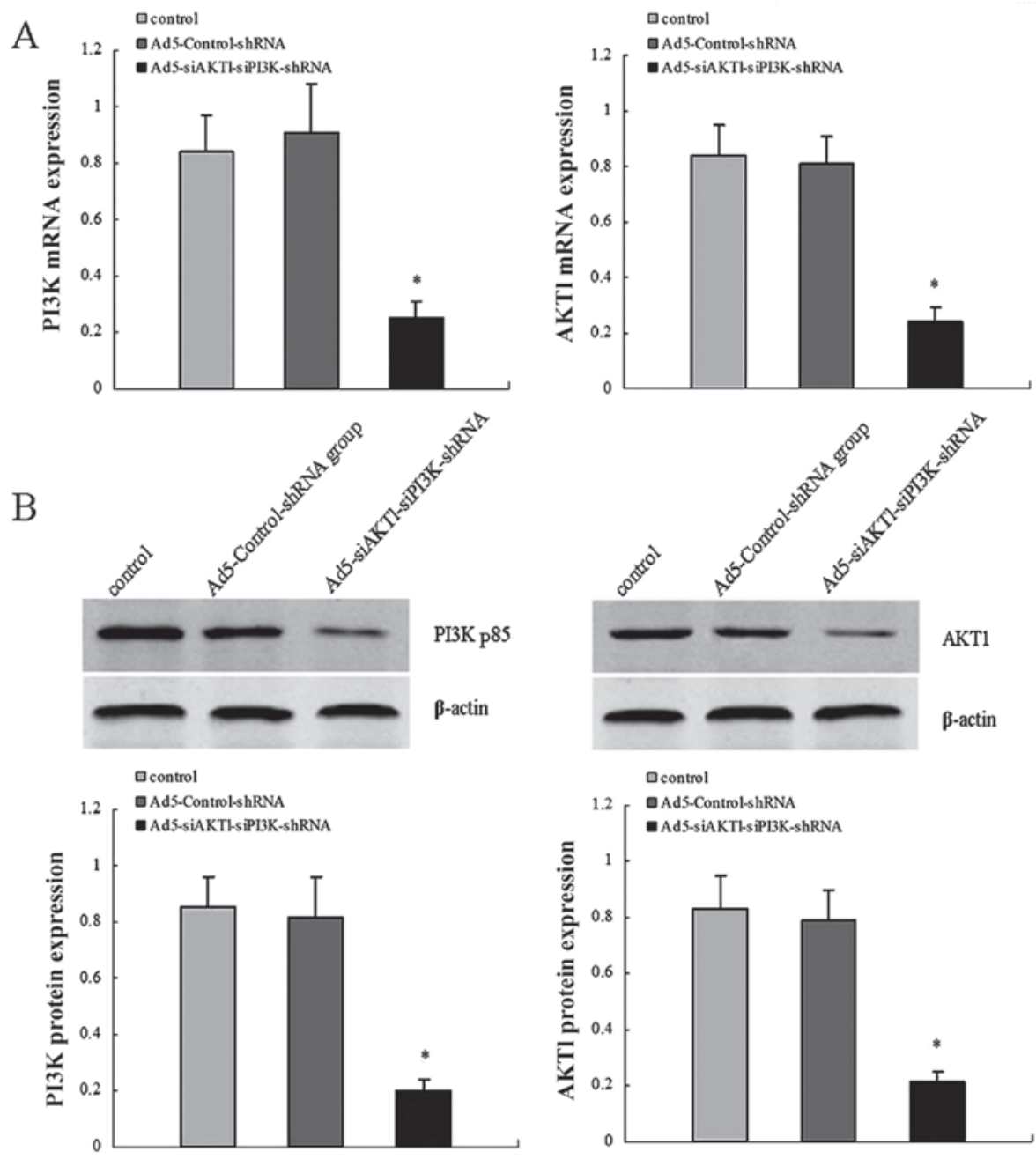

Figure 2. Reverse transcription-quantitative polymerase chain reaction and western blot assays revealed that the expression levels of AKT1 and PI3K/p85 (A) mRNA and (B) protein were significantly decreased in Ad5-siAKT1-siPI3K-shRNA group, compared with the control group or Ad5-Control-shRNA group. ("P<0.01, n=3). shRNA, short hairpin RNA; si, small interfering; PI3K, phosphatidylinositol 3-kinase.

field image of the same cell population. RT-qPCR and western blot assays were performed to detect AKT1 and PI3K/p85 mRNA and protein levels in control, Ad5-Control-shRNA and Ad5-siAKT1-siPI3K-shRNA groups. The results showed that the expression levels of AKT1 and PI3K/p85 mRNA and protein were significantly decreased in the Ad5-siAKT1-siPI3K-shRNA group, compared with the control or Ad5-Control-shRNA groups. The results demonstrated the high knockdown efficiency of Ad5-siAKT1-siPI3K-shRNA in vitro (Fig. 2).

PCNA, cyclin D1 and p53 protein expression in control, Ad5-Control-shRNA and Ad5-siAKT1-siPI3K-shRNA groups. Western blot assays were performed to detect PCNA, cyclin D1 and p53 protein levels. In these assays, expression levels of PCNA and cyclin D1 were significantly decreased in the Ad5-siAKT1-siPI3K-shRNA group, compared with the control or Ad5-Control-shRNA groups. While expression of p53 was significantly increased in the Ad5-siAKT1-siPI3K-shRNA group, compared with control or Ad5-Control-shRNA groups (Fig. 3).

AKT1 and PI3K/p85 knockdown decreases the proliferation rate of LCSCs. The Ad5-siAKT1-siPI3K-shRNA group exhibited a low proliferation rate when compared with the rate in the control group or Ad5-Control-shRNA groups beginning the fifth day after seeding (Fig. 4).

AKT1 and PI3K/p85 knockdown induces the arrest of the cell cycle of LCSCS. The cell cycle distribution was assessed using flow cytometry. The results revealed a marked arrest in the $\mathrm{G}_{0} / \mathrm{G}_{1}$ phase in the Ad5-siAKT1-siPI3K-shRNA group relative to the control group or Ad5-Control-shRNA group $(67.15 \pm 3.31 \%$ vs. $57.89 \pm 2.86 \%$ and $60.22 \pm 2.29 \%$; $<<0.01$, respectively) (Fig. 5).

AKT1 and PI3K/p85 knockdown slows the sphere-forming ability of LCSCs. The control, Ad5-Control-shRNA and Ad5-siAKT1-siPI3K-shRNA groups were examined for their ability to form new spheres after being initially cultured as a single cell. After 10 days, $49 \pm 5.4$ and $51 \pm 4.9 \%$ of wells with a single cell derived from the control group and the Ad5-Control-shRNA group formed a novel set of spheres, while only $14 \pm 2.7 \%$ of wells with a single cell derived from the Ad5-siAKT1-siPI3K-shRNA group was able to form spheres. These results indicate that the downregulation of AKT1 and PI3K/p85 leads to the loss of self-renewal in LCSCs (Fig. 6). 


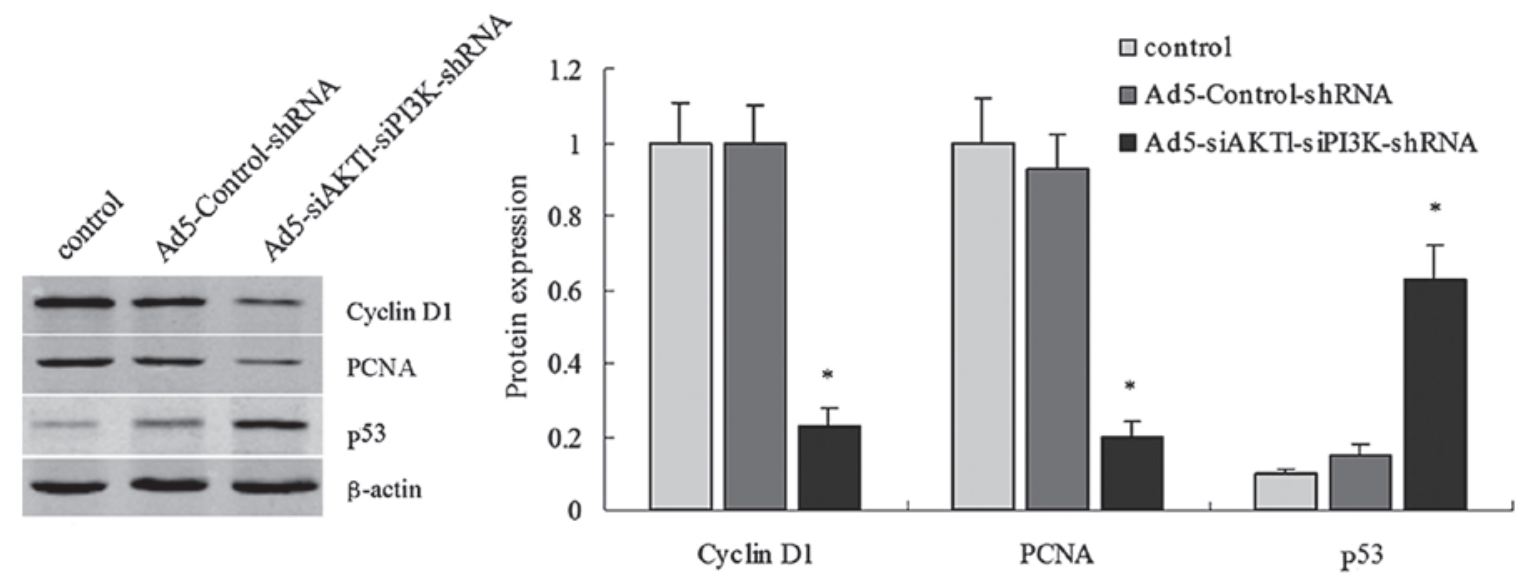

Figure 3. Western blot assays revealed that expression of PCNA and cyclin D1 were significantly decreased, while p53 was significantly increased following silencing of AKT1 and PI3K/p85 in LCSCs. ( ${ }^{*} \mathrm{P}<0.01, \mathrm{n}=3$ ). PCNA, ; shRNA, short hairpin RNA; si, small interfering; PI3K, phosphatidylinositol 3-kinase; proliferating cell nuclear antigen; LCSCs, lung cancer stem cells.

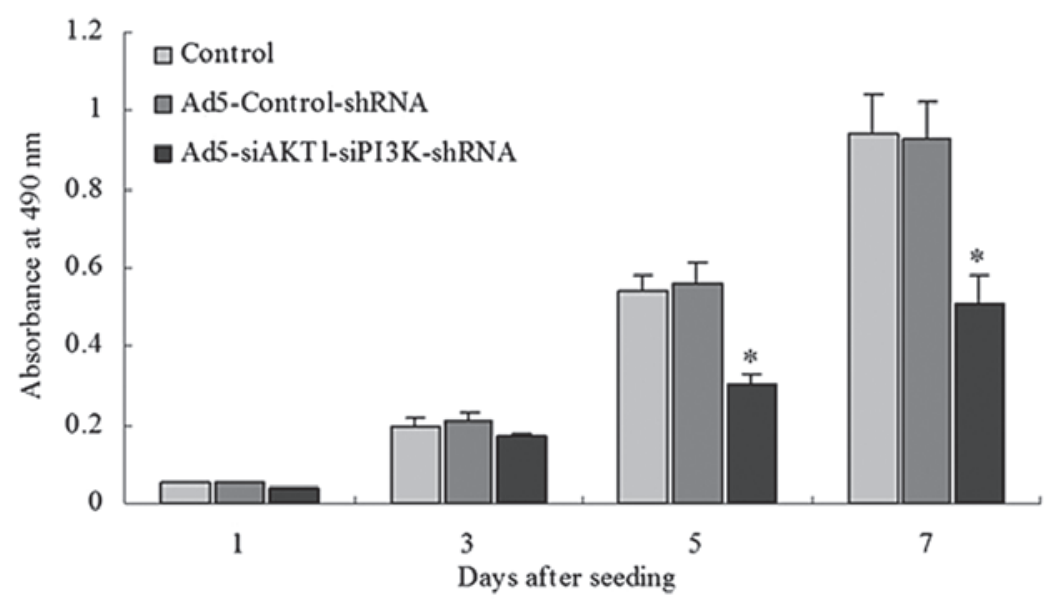

Figure 4. MTT proliferation assay revealed that lung cancer stem cells exhibited a low proliferation rate beginning the fifth day after silence of AKT1, PI3K/p85 ( $\mathrm{P}<0.01, \mathrm{n}=3$ ). shRNA, short hairpin RNA; si, small interfering; PI3K, phosphatidylinositol 3-kinase.
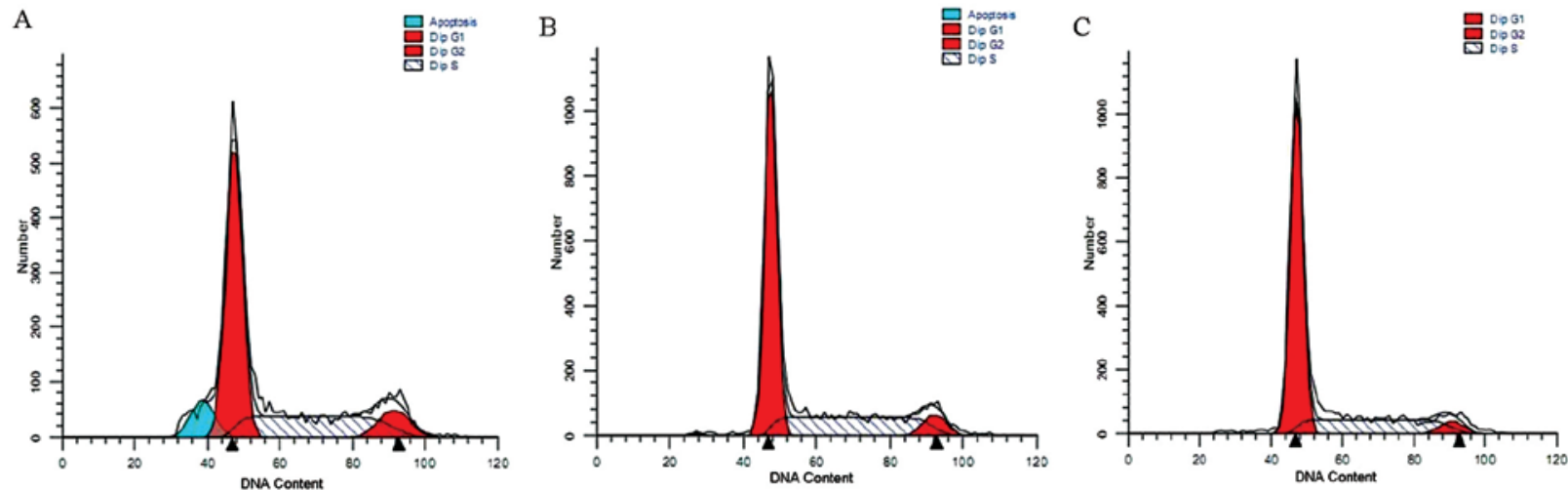

Figure 5. Epic XL flow cytometry instrument analysis revealed that the cell cycle of lung cancer stem cells were arrested in the $\mathrm{G}_{0} / \mathrm{G}_{1}$ phase following silencing of AKT1, PI3K/p85. (67.15 $\pm 3.31 \%$ vs. $57.89 \pm 2.86 \%$ and $60.22 \pm 2.29 \%, \mathrm{P}<0.01$, respectively). (A) control group, (B) Ad5-Control-shRNA group, (C) Ad5-siA KT1-siPI3K-shRNA group. shRNA, short hairpin RNA; si, small interfering.

AKT1 and PI3K/p85 knockdown decreases tumorigenic potential in LCSCS in NOD/SCID mice. The control, Ad5-Control-shRNA and Ad5-siAKT1-siPI3K-shRNA cells were injected subcutaneously into NOD/SCID mice in a limiting dilution experiment (i.e., $10^{2}, 10^{3}, 10^{4}$ and $10^{5}$ cells). The results showed that rAd5-siAKT1-siPI3K cells were associated with a decreased level of tumorigenicity relative to that of the control or Ad5-Control-shRNA groups. In order to 
Table I. Incidence of tumors of control group, Ad5-Control-shRNA group and Ad5-siAKT1-siPI3K-shRNA group cells serially transplanted into NOD/SCID mice.

\begin{tabular}{lllll}
\hline & \multicolumn{3}{c}{ Cell number } \\
\cline { 2 - 5 } Group & $10^{2}$ & $10^{3}$ & $10^{4}$ & $10^{5}$ \\
\hline Control & $2 / 5$ & $4 / 5$ & $5 / 5$ & \\
Ad5-Control-shRNA & $2 / 5$ & $4 / 5$ & $5 / 5$ & $3 / 5$ \\
Ad5-siAKT1-siPI3K-shRNA & $0 / 5$ & $0 / 5$ & $1 / 5$ & 3 \\
\hline
\end{tabular}

shRNA, short hairpin RNA; si, small interfering.

further compare the size of the xenograft tumor of the three groups in vivo, the xenograft tumor induced by $10^{5}$ cells in the three groups was observed and it was found that the tumor volume induced by the Ad5-siAKT1-siPI3K-shRNA group was also significantly lower than that induced by the control or Ad5-Control-shRNA group (Table I, Fig. 7).

\section{Discussion}

Current studies suggest that drug resistance mechanisms in tumor cells have a close association with CSCs, which may lead to resistance to radiation and chemotherapy through the following mechanisms: i) The majority of CSCs (such as leukemia stem cells and liver stem cells) are in the $\mathrm{G}_{0}$ phase. Due to their slow growth and primarily dormant state, CSCs are resistant to cell cycle-specific agents (20-22). ii) Various types of CSCs overexpress membrane $\mathrm{ABC}$ transporter and drug resistance genes, including P-glycoprotein, encoded by the $\mathrm{ABCB} 1$ gene, multidrug resistance-related protein 1 and breast drug resistance protein 2, encoded by the ABCG2/ MXR gene. These proteins can pump chemotherapeutic drugs out of the cell, decreasing the intracellular concentration and weakening their cytotoxic effects on cancer cells; thus, resulting in drug resistance (23-25). iii) CSCs express DNA repair protein and thymidylate synthase excision repair cross-complementation group 1 at a high level, increasing the capacity of cancer cells for repair following antitumor treatment, and thereby increasing resistance to anticancer drugs (26). iv) In comparison to normal stem cells, signaling pathways associated with self-renewal and proliferation in CSCs are often over-activated, becoming sources for tumor recurrence and metastasis $(27,28)$.

Although there have been few studies of the PI3K/AKT signaling pathway in stem cells, a previous study has confirmed that this pathway is important in the proliferation of breast cancer stem cells (18). Gong et al (29) found that the proliferation of osteosarcoma cancer stem cells was significantly decreased by the PI3K inhibitor LY294002 in a dose- and time-dependent manner, suggesting that the PI3K/AKT signaling pathway is an important mediator of osteosarcoma cancer stem cell proliferation. Targeted inhibition of this pathway can effectively inhibit the proliferation of cancer stem cells (29). Sunayama et al (30) used rapamycin in combination with LY294002 to treat glioma stem cells and showed that proliferation and the self-renewal capacity of these cells were

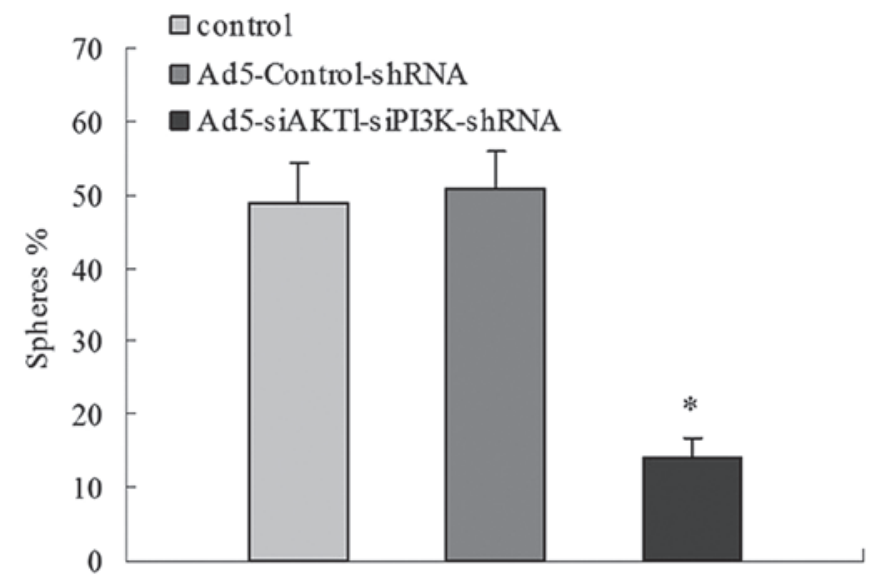

Figure 6. Sphere-forming assays revealed that Ad5-siAKT1-siPI3K-shRNA group cells exhibited decreased capacity for self-renewal, compared with control group or Ad5-Control-shRNA group ( $14 \pm 2.7$ vs. $49 \pm 5.4 \%$ or $51 \pm 4.9 \%,{ }^{*} \mathrm{P}<0.01, \mathrm{n}=3$ ). shRNA, short hairpin RNA; si, small interfering.

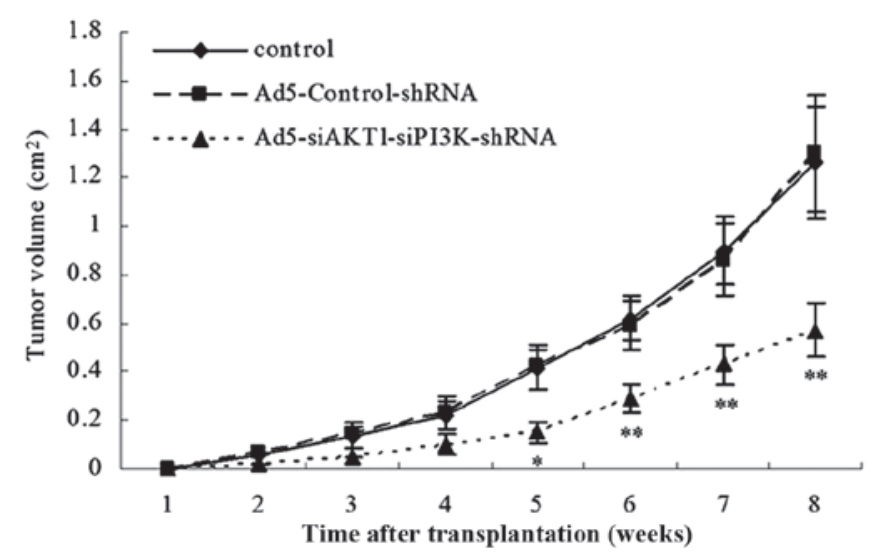

Figure 7.Xenograft tumor volume induced by $10^{5}$ cells of rAd5-siAKT1-siPI3K groups cells was significantly smaller than that of the same number of control group or Ad5-Control-shRNA group cells ( ${ }^{*} \mathrm{P}<0.05$ and ${ }^{* *} \mathrm{P}<0.01, \mathrm{n}=3$ ). shRNA, short hairpin RNA; si, small interfering.

diminished, as was tumorigenicity in vivo. Yang et al (19) successfully extracted bronchioloalveolar stem cells (BASCs) from a mouse model of K-ras-driven cancer. Following treatment with a PI3K/AKT pathway inhibitor, migration and proliferation of BASCs were reduced and the PTEN gene was inactivated. 
Currently, to the best of our knowledge, there are no reports demonstrating the involvement of the PI3K/AKT signaling pathway in the regulation of self-renewal, proliferation and differentiation of human LCSCs. In the present study, shRNA technology was used to downregulate the expression of AKT1 and the PI3K/p85 subunit in LCSCs and changes were detected in cell proliferation in three groups. LCSC proliferation slowed and the cell doubling time increased when AKT1 and PI3K/ p85 were silenced, suggesting that the PI3K/AKT signaling pathway promoted LCSC proliferation. In addition, the self-renewal capacity of LCSCs was analyzed through LCSC sphere formation. The results showed that the self-renewal capacity of LCSCs in the rAd5-siAKT1-siPI3K-shRNA group was lower than in the control group or Ad5-Control-shRNA group, indicating an important role for the PI3K/AKT signaling pathway in LCSC self-renewal.

To further investigate the mechanism by which the PI3K/AKT pathway controls LCSC proliferation, gene expression of PCNA, cyclin D1 and p53 was examined in three groups of LCSCs. PCNA is a nuclear protein closely associated with cell proliferation, while p53 was the first identified tumor suppressor gene. Diminished or absent expression of p53 is an important mechanism for promoting tumorigenesis. PCNA and p53 are closely related to each other. p53 protein can induce the synthesis of p21 and GAPD45 protein, which binds to, inactivates and degrades PCNA (31). Cyclin D1 is highly expressed in a number of different cancer cells, promoting the $\mathrm{G}_{0} / \mathrm{S}$ transition and stimulating tumor growth (32). In the present study, downregulation of AKT1 and $\mathrm{PI} 3 \mathrm{~K} / \mathrm{p} 85$ expression was accompanied by decreased expression of cyclin D1 and PCNA, and increased expression of p53. These results show that silencing of AKT1 and $\mathrm{PI} 3 \mathrm{~K} / \mathrm{p} 85$ can result in cell cycle arrest at the $\mathrm{G}_{0} / \mathrm{G}_{1}$ phase, while $\mathrm{S}$ phase cells are significantly decreased, resulting in inhibition of cell proliferation.

The present study also showed that LCSC tumorigenicity in the rAd5-siAKT1-siPI3K-shRNA group was significantly reduced, compared with the control groups or Ad5-Control-shRNA group, but was still present in the treated cells, implying that LCSCs retained part of their self-renewal capacity when AKT1 and PI3K/p85 had been silenced. It was speculated that silencing of AKT1 and PI3K/p85 blocks LCSCs from entering the cell cycle, inhibits the self-renewal rate of LCSCs and reduces the number of stem cells with tumorigenic properties by causing asymmetric division.

In conclusion, AKT1 and PI3K were overexpressed in LCSCs. Silencing of AKT1 and PI3K/p85 gene transcription reduced cell proliferation, tumor sphere formation and tumor development in NOD/SCID mice. This study provides a potential novel therapeutic strategy for the treatment of drug resistance and recurrence of lung cancer.

\section{References}

1. Zhou XD, Wang XY, Qu FJ, et al: Detection of cancer stem cells from the C6 glioma cell line. J Int Med Res 37: 503-510, 2009.

2. Qiu B, Zhang D, Tao J, Wu A and Wang Y: A simplified and modified procedure to culture brain glioma stem cells from clinical specimens. Oncol Lett 3: 50-54, 2012.

3. Hwang-Verslues WW, Kuo WH, Chang PH, et al: Multiple lineages of human breast cancer stem/progenitor cells identified by profiling with stem cell markers. PLoS ONE 4: e8377, 2009.
4. Collins AT, Berry PA, Hyde C, Stower MJ and Maitland NJ: Prospective identification of tumorigenic prostate cancer stem cells. Cancer Res 65: 10946-10951, 2005.

5. O'Brien CA, Pollett A, Gallinger S and Dick JE: A human colon cancer cell capable of initiating tumour growth in immunodeficient mice. Nature 445: 106-110, 2007.

6. Suetsugu A, Nagaki M, Aoki H, Motohashi T, Kunisada T and Moriwaki $\mathrm{H}$ : Characterization of $\mathrm{CD} 33^{+}$hepatocellular carcinoma cells as cancer stem/progenitor cells. Biochem Biophys Res Commun 351: 820-824, 2006.

7. Zhang DG, Jiang AG, Lu HY, Zhang DG, Zhang LX and Gao XY: Isolation, cultivation and identification of human lung adenocarcinoma stem cells. Oncol Lett 9: 47-54, 2015.

8. Varnat F, Duquet A, Malerba M, et al: Human colon cancer epithelial cells harbour active HEDGEHOG-GLI signalling that is essential for tumour growth, recurrence,metastasis and stem cell survival and expansion. EMBO Mol Med 1: 338-351, 2009.

9. Sullivan JP, Minna JD and Shay JW: Evidence for self-renewing lung cancer stem cells and their implications in tumour initiation, progression and targeted therapy. Cancer Metastasis Rev 29: $61-72,2010$

10. Meng M, Zhao XH, Ning Q, Hou L, Xin GH and Liu LF: Tumor stem cells:A new approach for tumor therapy (Review). Oncol Lett 4: 187-193, 2012.

11. Xu Y, Hu YD, Zhou J, Zhang MH, Yuan WW and Luo Y: shRNA targeting Bmil impedes the self-renewal of cisplatin-enriched stem-like cells in human A549 cells. Oncol Rep 28: 629-639, 2012.

12. Cantrell DA: Phosphoinositide 3-kinase signaling pathways. J Cell Sci 114: 1439-1445, 2001.

13. Hennessy BT, Smith DL, Ram PT, Lu Y and Mills GB: Exploiting the PI3K/AKT pathway for cancer drug discovery. Nat Rev Drug Discov 4: 988-1004, 2005.

14. Sun XY, Ding LS and Jin XD, et a1: Correlation of PI3K/Akt/mTOR signal transduction pathway with both malignancy progression and prognosis of human gliomas. Chin J Neuromed 10: 24-28, 2011 (In Chinese).

15. Missiaglia E, Dalai I, Barbi S, et al: Pancreatic endocrine tumors:expression profiling evidences a role for AKT-mTOR pathway. J Clin Oncol 28: 245-255, 2010.

16. Jiang AG, Yu H and Huang JA: Expression and clinical significance of the PI3K/Akt signal transduction pathway in non-small cell lung carcinoma. Oncol Lett 8, 601-607, 2014.

17. Sunayama J, Sato A, Matsuda K, et al: Dual blocking of mTor and PI3K elicits a prodifferentiation effect on glioblastoma stem-like cells. Neuro Oncol 12: 1205-1219, 2010.

18. Zhou J, Wulfkuhle J, Zhang $\mathrm{H}$, et al: Activation of the PTEN/mTOR/STAT3 pathway in breast cancer stem-like cells is required for viability and maintenance. Proc Natl Acad Sci USA 104: 16158-16163, 2007.

19. Yang Y, Iwanaga K, Raso MG, et al: Phosphatidylinositol 3-kinase mediates bronchioalveolar stem cell expansion in mouse models of oncogenic k-ras-induced lung cancer. PLoS One 3: e2220, 2008.

20. Loebinger MR, Eddaoudi A, Davies D and Janes SM: Mesenchymal stem cell delivery of TRAIL can eliminate metastatic cancer. Cancer Res 69: 4134-4142, 2009.

21. Levi E, Misra S, Du J, Patel BB and Majumdar AP: Combination of aging and dimethylhydrazine treatment causes an increase in cancer-stem cell population of rat colonic crypts. Biochem Biophys Res Commun 385: 430-433, 2009.

22. Ghotra VP, Puigvert JC and Danen EH: The cancer stem cell microenvironment and anti-cancer therapy. Int J Radiat Biol 85: 955-962, 2009.

23. Ho MM, Ng AV, Lam S and Hung JY: Side population in human lung cancer cell lines and tumors is enriched with stem-like cancer cells. Cancer Res 67: 4827-4833, 2007.

24. Sung JM, Cho HJ, Yi H et al: Characterization of s stem cell population in lung cancer A549 cells, Biochem Biophys Res Commun 371: 163-167, 2008.

25. Levina V, Marrangoni AM, DeMarco R, Gorelik E and Lokshin AE: Drug-selected human lung cancer stem cells: cytokine network, tumorigenic and metastatic properties. PLoS One 3: e3077, 2008.

26. Ota S, Ishii G, Goto K, et al: Immunohistochemical expression of BCRP1 and ERCC1 in biopsy specimen predicts survival in advanced non-small-cell lung cancer treated with cisplatin-based chemotherapy. Lung Cancer 64: 98-104, 2009.

27. Smith KS, Chanda SK, Lingbeek M, et al: Bmi-1 regulation of INK4A-ARF is adownstream requirement for transformation of hematopoietic progenitors by E2a-Pbx1. Mol Cell 12: 393-400, 2003. 
28. Lessard J and Sauvageau G: Bmi-1 determines the proliferative capacity of normal and leukaemic stem cells. Nature 423: 255-260, 2003.

29. Gong C, Guo FJ, Qin L, et al: LY294002 inhibits proliferation of cancer stem-like cells from human osteosarcoma via down-regulation of PI3K/AKT signaling pathwa. Chin J Exp Surg 28: 2215-2217, 2011 (In Chinese).

30. Sunayama J, Sato A, Matsuda K, et al: Dual blocking of mTor and PI3K elicits a prodifferentiation effect on glioblastoma stem-like cells. Neuro Oncol 12: 1205-1219, 2010.
31. Cayrol C, Knibiehler M and Ducommun B: p21 binding to PCNA causes G1 and G2 cell cycle arrest in p53-deficient cells. Oncogene 16: 311-320, 1998.

32. Lebeau A, Unholzer A, Amann G, et al: EGFR, HER-2/neu, cyclin D1, p21 and p53 in correlation to cell proliferation and steroid hormone receptor status in ductal carcinoma in situ of the breast. Breast Cancer Res Treat 79: 187-198, 2003. 